\title{
Erlebnis Stadtpark. Nutzung und Wahrnehmung urbaner Grünräume
}

\author{
S. Hennecke \\ Universität Kassel, Fachbereich Architektur Stadtplanung Landschaftsplanung (ASL), Fachgebiet \\ Freiraumplanung, Kassel, Germany \\ Correspondence to: S. Hennecke (hennecke@uni-kassel.de)
}

\begin{abstract}
Kaspar, H.: Erlebnis Stadtpark. Nutzung und Wahrnehmung urbaner Grünräume, Reihe Erlebniswelten, Herausgeber: Gebhardt, W., Hitzler, R., und Liebl, F., Wiesbaden, Springer Verlag für Sozialwissenschaften, 303 S., ISBN-13: 9783531 18715 0, € 32,99 Softcover oder $€ 24,99$ als E-Book, 2012.
\end{abstract}

Öffentliche Parkanlagen sind ein hoch geschätzter Bestandteil europäisch geprägter Städte. Die Geschichte des Stadtparkes ist im Vergleich zur Geschichte der Gartenkunst relativ jung. Stadtparkanlagen sind ein Ergebnis der Industrialisierung und des Stadtwachstums im 19. Jahrhundert. Sie wurden von Anfang an von denjenigen, die sie projektierten oder über sie schrieben, als Orte der Erholung von den Zumutungen städtischen Lebens und als Orte sozialer Integration konzipiert. Heute finden sich als Begründung für die Planung neuer Parkanlagen noch ganz ähnliche Argumente wie vor zweihundert Jahren. Angesichts der wichtigen Rolle, die Stadtparkanlagen im städtischen Raum- und Sozialgefüge zugeschrieben wird, findet man erstaunlich wenige wissenschaftliche Untersuchungen dazu, ob diese Räume die ihnen zugedachte Rolle auch tatsächlich erfüllen.

Das Buch „Erlebnis Stadtpark. Nutzung und Wahrnehmung urbaner Grünräume“ von Heidi Kaspar aus Zürich leistet einen wichtigen und hochinteressanten Beitrag zur Behebung dieses Forschungsdefizits. Die Studie entstand als Promotion am Geografischen Institut der Universität Zürich und wurde - betreut von Elisabeth Bühler - im Rahmen des vom Schweizer Nationalfonds geförderten Forschungsprojektes „Nachhaltige Parkanlagen“ erstellt. Aus der Perspektive der Sozialgeografie untersucht Heidi Kaspar das individuelle Erleben der Nutzerinnen und Nutzer kleinerer städtischer Parkanlagen in Zürich. Ihr Ziel ist es, die jeweils subjektive Konstitution von Park-Räumen zu rekonstruieren und daraus Schlüsse für die praktische Planung von öffentlichen Grünräumen zu ziehen. Dem liegt die Aus- gangsthese zugrunde, dass städtische Grünräume „erst durch Wahrnehmungs-, Vorstellungs- und Erinnerungsprozesse sowie (durch die) darauf beruhenden alltäglichen Praktiken zu eben diesen Park-Räumen (werden) - mit den spezifischen Eigenschaften derentwegen der Ort aufgesucht oder aber gemieden wird" (S. 14).

Der Stadtpark wird von Heidi Kaspar als unspektakulärer Ort des alltäglichen Lebens treffend mit der Aussage einer von ihr befragten Parknutzerin charakterisiert: „Es ist nichts Besonderes, eigentlich. Also für mich schon.“ (S. 17). Kaspar will diese gesellschaftliche und dennoch nur individuell beschreibbare Bedeutung von Parkräumen für die Nutzerinnen und Nutzer aufspüren und verallgemeinerbar beschreiben. Der Erlebnisraum Stadtpark wird dabei zu Beginn sehr treffend gegen andere „Erlebniswelten“ - so der Titel der Reihe, in der dieses Buch herausgegeben wurde - wie etwa Freizeit- oder Vergnügungsparks abgegrenzt werden. Stadtparks zeichnen sich, so ein Fazit des Buches, gerade durch ihre „Alltäglichkeit und Unaufgeregtheit“ (S. 57) aus und es gilt, genau diese spezifischen Eigenschaften zu erhalten (S. 268).

Eine große Qualität von Kaspars Studie ist es, dass die Autorin das eigene Vorgehen bei der empirischen Untersuchung und der Interpretation des gesammelten Datenmaterials durchgehend reflektiert. Auch ihr eigener Einfluss als Forscherin auf die Untersuchungsergebnisse wird laufend diskutiert. So stellt das Buch neben den besonders auch für die Planungspraxis sehr aufschlussreichen Ergebnissen eine Art Lehrbuch für die „explorative und praxiszentrierte“ (S. 26). Auseinandersetzung mit Fragen der Wahrnehmung und Nutzung öffentlicher Freiräume dar.

Das Buch gliedert sich in vier große Abschnitte mit zwölf inhaltlichen Kapiteln sowie einem Anhang.

$\mathrm{Zu}$ Beginn führt Kaspar in den Stand der Forschung zu Parkanlagen als Natur-Landschaften, als Teilbereich der städtischen Öffentlichkeit und als alltägliche Erlebniswelten 
ein (Kapitel 2) und verortet ihr eigenes Forschungsvorhaben im Kontext der Raumtheorie von Martina Löw. Sie verbindet dies mit dem überzeugend dargelegten Anspruch diese Raumtheorie um den Begriff des „Erlebens“ zu erweitern. Das Erleben schließt, so Kaspar, die für ihre Untersuchung wichtigen sinnlichen, körperlichen und emotionalen Komponenten mit ein, die im Prozess der Konstruktion von Parkräumen eine entscheidende Rolle spielen (S. 80). Einen zweiten für die Arbeit zentralen theoretischen Bezugspunkt stellt die Diskussion um die Konstruktion von Geschlecht dar. Die Herstellung von Geschlecht in der alltäglichen Interaktion wird von Kaspar als wichtiger Subtext jeder Aushandlung von Nutzungsansprüchen im öffentlichen Raum - und so auch in den von ihr untersuchten Parkräumen - thematisiert (Kapitel 3). Besonders interessiert sie dabei der öffentlich geführte Diskurs über die Gefährdung von Frauen im öffentlichen Raum, der - von ihr in diesem Ausmaß nicht erwartet - eine große Bedeutung für die Nutzung oder eben gerade die Nicht-Nutzung der Parkanlagen durch Frauen hat. Kaspar spricht vom Selbstausschluss der Parknutzerinnen aus diesen Räumen, die diese mit der rein hypothetischen Erwartung von sexuellen Übergriffen begründen (Kapitel 7). Die parallele Diskussion der Konstruktion von Raum und Geschlecht im Rahmen dieser Untersuchung eröffnet theoretisch ergiebige Perspektiven, um die beiden getrennt voneinander geführten Diskurse zu verknüpfen.

Der zweite Abschnitt des Buches ist der Darlegung der Untersuchungsmethode und der Einführung in die drei untersuchten Parkanlagen im Zürcher Stadtgebiet gewidmet. Den zentralen Datenkorpus bilden leitfadengestützte ausführliche Interviews mit Nutzerinnen und Nutzern der drei Parkanlagen, die zu verschiedenen Tageszeiten geführt wurden. Kaspar legt detailliert dar, wie sie die Auswahl der Interviewten getroffen und den Interviewleitfaden so strukturiert hat, dass im Interview genau dasjenige, was von den Befragten für unbedeutend und alltäglich gehalten und daher nicht angesprochen wird, thematisiert werden kann. In den ausführlichen Zitaten aus den Transskripten der Interviews wird deutlich, welche große Herausforderung es darstellt, das scheinbar Banale und doch für das allägliche Leben Bedeutsame in Worte zu fassen: „Ja es ist immer schwierig, weil ich weiß nicht, meistens hat's einfach viele Leute hier, die ich einfach kenne und dann gehe ich zu ihnen hin und dann sitzen wir und dann irgendwann gehe ich wieder nach Hause. (...) Also wir machen dann eigentlich nichts wirklich hier. Wir sind einfach hier." (Interviewtransskript S. 143).

Der dritte Abschnitt des Buches besteht in der Darstellung der Interviewauswertung mit der Methode der Grounded Theory aus der qualitativen Sozialforschung. Sehr gut nachvollziehbar und reich an interessanten Einzelaspekten wird das Datenmaterial interpretiert. Dabei werden das Erleben des Geschehens im Park und das Erleben der ParkGestalt zunächst getrennt voneinander betrachtet. Zentral, so Kaspar, für einen Besuch im Park ist die Anwesenheit anderer Menschen in diesen öffentlichen Räumen. Der Park dient als Treffpunkt mit Bekannten aber auch als Ort, an den man sich gezielt in die Gesellschaft Unbekannter begibt. Die anderen Parknutzerinnen und Parknutzer gehören zum Stadtparkerlebnis ebenso dazu wie die unbeweglichen Raumelemente, die Bänke, Bäume, Rasenflächen. Kaspar beschreibt den Stadtpark als Ort der Aushandlung von Nutzungsansprüchen, als Ort sozialer Zumutungen aber auch sozialer Integration (Kapitel 6, 7 und 8). Sie spricht von der „Zartheit sowohl des harmonischen Nebeneinanders als auch des Rechts auf den öffentlichen Raum“, das besonders auch durch die fortwährende gegenseitige Zuschreibung von (Geschlechter)-Rollen und die damit verbundene Reproduktion von gesellschaftlichen Hierarchien in Frage gestellt werde (S. 197).

Besonders interessant aus der Perspektive von Planerinnen und Planern und Entwerferinnen und Entwerfern, deren Aufgabe darin besteht, neue Parkanlagen zu konzipieren, ist Kaspars Untersuchung der Bedeutung der gebauten und gepflanzten Gestalt der Parkanlagen für deren Nutzerinnen und Nutzer. Hier eröffnet die Studie eine anregende Perspektive für die Zusammenführung sozialwissenschaftlicher Forschung und Planungs- sowie Entwurfspraxis. Die Bedeutung der Gestalt von Parkanlagen tritt durch die kontrastierende Auswahl von drei sehr unterschiedlich gestalteten Parkanlagen besonders deutlich hervor. Kaspar befragte Nutzerinnen und Nutzer in einer in der Tradition von Stadtparkanlagen aus dem 19. und frühen 20. Jahrhundert als ,klassisch“ zu bezeichnenden Parkanlage (Bäckeranlage), einer als ,naturnah“ gestaltet einzuordnenden Anlage von 1989 mit einfacher Ausstattung und einfachem Pflegestandard (SaveraAreal) und einer erst wenige Jahre alten Parkanlage in einem neuen Stadtentwicklungsgebiet Zürichs, deren Gestaltungssprache als ,,architektonisch“ beschrieben werden kann (Wahlenpark). Der Wahlenpark als Untersuchungsort ist für die Landschaftsarchitektur von besonderem Interesse, da hier die positive Resonanz in der Fachpresse sehr stark von der negativen Resonanz auf die gebaute Parkanlage in der lokalen Tagespresse abweicht (S. 139). Die ersten beiden Parks gehen aus den Ergebnissen der Untersuchung als in der Regel gut nutzbar hervor, da sie in ihrer Gestaltung die Erwartungen der Nutzerinnen und Nutzer an eine Parkanlage weitgehend erfüllen. Aber, so Kaspar, damit reproduzieren sie auch die bestehenden sozialen Konventionen in der Aushandlung von Nutzungsansprüchen, etwa bei der intuitiven Abgrenzung von Orten für Spiel, für das Lagern, für den ruhigen Rückzug oder für den Aufenthalt sozialer Randgruppen. Der Wahlenpark bietet demgegenüber in seiner Gestaltung wenig Anknüpfungspunkte für die Erwartungen an einen ,normalen“ Stadtpark. Er schließt damit, so ein Ergebnis der Untersuchung, Nutzerinnen und Nutzer alleine deshalb aus, weil er für sie nicht als Park lesbar ist (Kapitel 10). Allerdings, und das macht die erfreuliche Differenziertheit der Studie von Kaspar aus, wird der Wahlenpark genau wegen dieser Unlesbarkeit auch als Ort möglicher Emanzipation von sozialen Konventionen interpretiert. Die ,,semantische Unbe- 
stimmtheit" einzelner Elemente im Wahlenpark erlaube unerwartete Nutzungen und provoziert vor allem auch durch deren sonst nicht übliches Nebeneinander neue Notwendigkeiten zur Aushandlung von Raumnutzung. Kaspar erkennt daher ein „,utopisches Moment“ in einer Gestaltung, die gewohnte Formen hinter sich lässt, und würdigt deren möglichen Beitrag zu „emanzipatorischen und integrativen Räumen“" (S. 266).

Dieses für die Landschaftsarchitektur ermutigend klingende Fazit kann aber nicht als Freibrief zur kontextlosen und freien Formgebung gelesen werden. Vielmehr kommt diese Studie zu dem Schluss, dass ein Park nicht ein eindeutig lesbarer Raum ist, sondern aus genau so vielen Räumen zusammengesetzt ist, die im tagtäglichen Gebrauch und immer wieder aufs Neue von den unterschiedlichsten Nutzerinnen und Nutzern konstituiert werden. Daher ist die Konzipierung, Planung, Gestaltung und Unterhaltung von Parkanlagen eine gesellschaftlich ungemein verantwortungsvolle und komplexe Aufgabe. Das emanzipatorische Potential von Gestaltung ist dabei nur ein Baustein. Und genau das macht - nach der unbedingt empfehlenswerten Lektüre dieses Buches - auf die interdisziplinäre Zusammenarbeit derjenigen, die Parkanlagen planen und gestalten mit denjenigen, die sich mit dem Geschehen in Parkanlagen wissenschaftlich auseinandersetzen, und nicht zuletzt mit denjenigen, die Parkanlagen in ihrem Alltag erleben, neugierig. 